\title{
Boundary driven unconventional mechanism of macroscopic magnetic field generation in beam-plasma interaction
}

\author{
Amita Das $\odot,{ }^{1, *}$ Atul Kumar $\odot,{ }^{2,3, \dagger}$ Chandrasekhar Shukla, ${ }^{2,3}$ Ratan Kumar Bera $\odot,{ }^{2,3}$ Deepa Verma $\odot,{ }^{2,3}$ \\ Devshree Mandal $\odot,{ }^{2,3}$ Ayushi Vashishta $\odot,{ }^{2,3}$ Bhavesh Patel, ${ }^{2}$ Y. Hayashi $\odot,{ }^{4}$ K. A. Tanaka $\odot,{ }^{4,5}$ G. Chatterjee, ${ }^{6}$ \\ Amit D. Lad ${ }^{6}{ }^{6}$ \\ G. Ravindra Kumar $\odot, 6,8$ and Predhiman $\mathrm{Kaw}^{2, \sharp}$ \\ ${ }^{1}$ Department of Physics, Indian Institute of Technology, Delhi, Hauz Khas, New Delhi 110016, India \\ ${ }^{2}$ Institute for Plasma Research, Bhat, Gandhinagar 382428, India \\ ${ }^{3}$ Homi Bhabha National Institute, Mumbai 400094, India \\ ${ }^{4}$ Graduate School of Engineering, Osaka University, Suita, Osaka 565-0871, Japan \\ ${ }^{5}$ Extreme Laser Infrastructure-Nuclear Physics, 30 Reactorului, Bucharest, Magurele 077125, Romania \\ ${ }^{6}$ Tata Institute of Fundamental Research, 1 Homi Bhabha Road, Mumbai 400005, India
}

(Received 1 May 2019; revised 3 October 2019; accepted 3 August 2020; published 14 September 2020; corrected 5 October 2020)

\begin{abstract}
The all pervading magnetic field in nature has aroused great curiosity and spawned many efforts to understand its generation. We propose, simulate, and experimentally demonstrate another mechanism of long-scale magnetic field generation in the context of a laser-plasma interaction. It relies on two realistic features, namely the finite size of the laser generated electron beam and an initial current imbalance. It is shown that magnetic fields of scale lengths comparable to the transverse beam dimension, are generated much before the onset of conventional instabilities associated with the beam-plasma system. This is due to radiative leakage at the boundaries of the finite beam, wherein even a small but finite current imbalance plays the crucial role of a radiative antenna. These features have been absent in simulations and theoretical analyses using the periodic boundary condition.
\end{abstract}

DOI: 10.1103/PhysRevResearch.2.033405

The dynamical evolution of intense magnetic fields and associated current pulses plays an important role in a variety of plasma physics problems. These include plasma switches, the design of novel radiation and charged particle sources, laser driven fusion, laboratory simulation of astrophysical phenomena, etc. [1-7]. Current pulses may be generated and driven into a solid density plasma by an intense femtosecond laser. It is now well known that massive current pulses produced in such an interaction induce return currents from the thermal plasma electrons, and these two types of currents are fraught with electromagnetic instabilities. It is widely believed that the well-known Weibel instability [8-10] separates these initially superimposed counterstreaming currents and leads to strong magnetic field generation. Countless analytical and simulation studies have explored this instability and predicted that this instability generates a magnetic field at the electron skin depth scale. The nonlinear inverse cascade mechanism subsequently leads to the progressive generation of longer scales $[9,11-14]$.

\footnotetext{
*amitadas3@yahoo.com

†atul.j1211@gmail.com

†Deceased.

§grk@tifr.res.in
}

Published by the American Physical Society under the terms of the Creative Commons Attribution 4.0 International license. Further distribution of this work must maintain attribution to the author(s) and the published article's title, journal citation, and DOI.
In this paper, we provide convincing evidence that this is not a realistic physical situation but merely an artifact of a boundless beam-plasma interaction in simulations as well as theoretical analyses. We demonstrate through laser-plasma experiments and finite beam size simulations, using different varieties of codes, that the energy is input preferentially into the magnetic field at the scale of the beam (in the case of laser experiments this is at the spot size of the irradiating laser). We show that magnetic field generation caused by the electron currents first occurs at the scale of the boundary and only much later does the Weibel instability kick in at the skin depth scale. This is another mechanism of magnetic field generation which is triggered by a small current imbalance acting as an antenna causing radiative leakage from the two edges of the finite beam. When the plasma medium is overdense, the radiatively leaking fields accumulate at the beam edge, giving rise to long-scale magnetic fields. This mechanism of magnetic field generation dominates the Weibel instability at the initial stage and has its origin in the effect of radiative loss from negative energy waves in a manner analogous to the radiative instability of a leaky waveguide $[15,16]$.

We have carried out theoretical analyses using a two-fluid model for an equilibrium configuration of the beam-plasma system in a two-dimensional (2D) $x-y$ plane as shown in Fig. 1(a). The central region II from $-a \leqslant y \leqslant a$ carries the beam current and an oppositely flowing background plasma current, which balance each other. In regions I and III the plasma is static and at rest. The charge neutralization in equilibrium is achieved by balancing the total electron density by the background ion density, viz., $\sum_{\alpha} n_{0 \alpha}=n_{0 i}$ in all three 
(a)

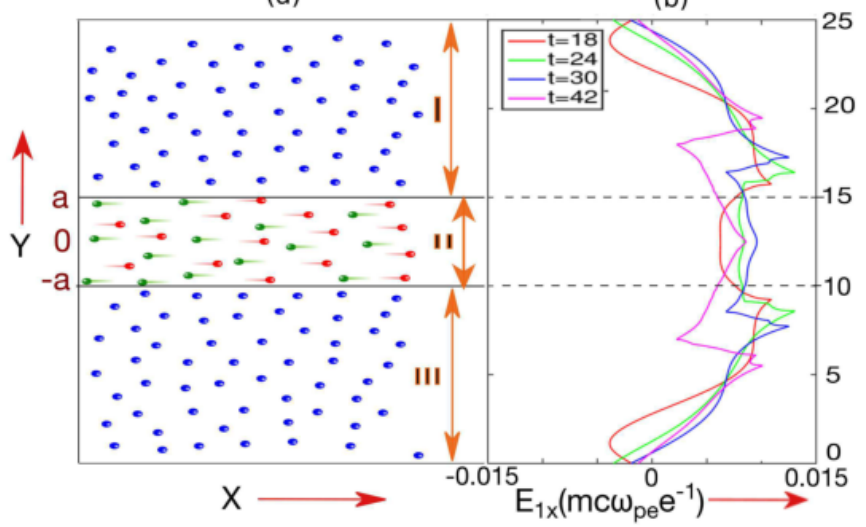

FIG. 1. (a) Schematics of 2D equilibrium geometry of the beamplasma system where the beam has a finite width $2 a$ in the transverse direction, and (b) mean electric field $\left(E_{1 x}\left[m c \omega_{p e} e^{-1}\right]\right)$ profile as a function of $y\left(c / \omega_{p e}\right)$ from 2D PIC simulations have been shown at various times. It is noteworthy that the variation of $E_{1 x}$ is minimal in the bulk region $\left(y=10-15 c / \omega_{p e}\right)$.

regions. The electron flow velocity in regions I and III is zero, whereas in region II the two fluids flow in a fashion so as to have zero current, i.e., $\sum_{\alpha} n_{0 \alpha} v_{0 \alpha x}=0$. Here, the suffix $\alpha$ stands for $b$ and $p$ representing the beam and plasma electrons and $v_{0 \alpha}$ are equilibrium flow velocities of the species in region II as shown in Fig. 1(a). The linearized perturbation of the momentum and continuity equations of the two fluids and the Maxwell set of equations around this equilibrium are considered. The perturbed fields are chosen to vary with respect to $y$ and $t$ only. The flow is confined in the $x-y$ plane, so we have $B_{1 z}, E_{1 x}$, and $E_{1 y}$ (where the suffixes $x, y$, and $z$ denote the spatial components and $B$ and $E$ represent the magnetic and electric field, respectively) only as the perturbed dominant fields. Taking the Fourier transform in time and eliminating all the perturbed fields in terms of $E_{1 x}$ leads to the following differential equation,

$$
\left[f_{2} E_{1 x}^{\prime}\right]^{\prime}-g_{2} E_{1 x}=0 .
$$

Here, $f_{2}=1+S_{4} / \omega^{2}-S_{3}^{2} /\left[\omega^{2}\left(S_{1}-\omega^{2}\right)\right], \quad g_{2}=S_{2}-\omega^{2}$, and the prime symbol ' denotes differentiation with respect to $y$. Furthermore, $S_{1}=\sum_{\alpha} n_{0 \alpha} /\left(n_{0} \gamma_{0 \alpha}\right), S_{2}=\sum_{\alpha} n_{0 \alpha} /\left(n_{0} \gamma_{0 \alpha}^{3}\right)$, $S_{3}=\sum_{\alpha}\left(n_{0 \alpha} v_{0 x \alpha}\right) /\left(n_{0} \gamma_{0 \alpha}\right)$, and $S_{4}=\sum_{\alpha}\left(n_{0 \alpha} v_{0 x \alpha}^{2}\right) /\left(n_{0} \gamma_{0 \alpha}\right)$; here, $\omega=\omega_{r}+i \gamma$ is the mode frequency with $\omega_{r}$ and $\gamma$ as the real and imaginary parts of it, respectively, and $\gamma_{0 \alpha}$ is the Lorentz factor of the $\alpha$ species. It should be noted that $S_{3}$ and $S_{4}$ are finite only when there is an equilibrium flow in the two-fluid electron depiction. Moreover, if the two electron fluids have an identical density and equal and opposite flow velocities, then $S_{3}=0$. We now seek the possibilities for obtaining purely growing modes in a finite system. For this purpose, we multiply Eq. (1) by $E_{1 x}$, replace $\omega^{2}=-\gamma^{2}$ (for purely growing modes), and integrate over $y$ over region II, i.e., from $-a$ to $a$. This yields

$$
\int_{-a}^{a}\left[E_{1 x}\left(f_{2} E_{1 x}^{\prime}\right)^{\prime}-g_{2} E_{1 x}^{2}\right] d y=0,
$$

Upon integrating Eq. (2) by parts and observing that in region II, $f_{2}$ and $g_{2}$ are constant, we obtain

$$
\left.f_{2}\left[E_{1 x} E_{1 x}^{\prime}\right]\right|_{-a} ^{a}-f_{2} \int_{-a}^{a}\left[E_{1 x}^{\prime}\right]^{2} d y-g_{2} \int_{-a}^{a} E_{1 x}^{2} d y=0 .
$$

If the boundary term is absent, as in the case of an infinite homogeneous system, then Eq. (3) can be satisfied for a finite value of $E_{1 x}$, provided the second and third terms have opposite signs. The integrand being positive definite, this is possible provided $g_{2}$ and $f_{2}$ have opposite signs. The definitions of $g_{2}$ and $f_{2}$ in terms of $\gamma^{2}$ are $f_{2}=1+$ $S_{3}^{2} /\left[\gamma^{2}\left(S_{1}+\gamma^{2}\right)\right]-S_{4} / \gamma^{2}$ and $g_{2}=S_{2}+\gamma^{2}$. Since $g_{2}$ is positive, the only possible way for $f_{2}$ to be negative is to have $S_{4} / \gamma^{2}$ dominate over the first two terms of $f_{2}$. Thus, the conventional Weibel gets driven by $S_{4}$. It is also obvious that $S_{3}$ provides a stabilizing contribution, making it more difficult for $f_{2}$ to become negative which is desired for Weibel destabilization [9].

For a finite system, an interesting thing happens when the boundary contribution is retained. The boundary term $\left.\left[E_{1 x} E_{1 x}^{\prime}\right]\right|_{-a} ^{a}$ represents the Poynting flux and is positive for the radiative flux moving outside region II. The sign of the boundary term thus gets determined by the sign of $f_{2}$. Hence, another possibility for satisfying Eq. (3) with a finite and positive value of the first term balancing the other two negative terms exists.

It should be noted that the disturbances in this case are different from the Weibel mode as the boundary terms are responsible for it and the sign of $f_{2}$ is positive, which is different from the Weibel case. Furthermore, it is interesting to observe that Eq. (3) can be easily satisfied by the boundary contribution provided the variations in $E_{1 x}$ in the bulk are minimal as this leads to an insignificant contribution from the integrand of the second term. Thus the instability driven by the boundary term would have a preference for long-scale excitation. It is clear that for this mechanism to be operative, a finite beam with boundaries is essential. Also, the radiative outward flux needs to be triggered.

We now show evidence of long-scale magnetic field structures that are generated via this mechanism. We provide this through numerical simulations as well as experimental observations. The particle-in-cell (PIC) simulations under OSIRIS 4.0 framework were carried out in both $2 \mathrm{D}$ [results depicted in Figs. 1(b), 2, and 4] and 3D (Fig. 3). The 2D simulation geometry is exactly the same as the one used in our analysis. In $3 \mathrm{D}$ a solid cylindrical beam current of diameter $2 a$ was chosen. The 2D simulations were carried for a box size of $25 c / \omega_{p e} \times 25 c / \omega_{p e}$. The beam was chosen to be confined within an extent of $2 a=5 c / \omega_{p e}$. The spatial resolution is taken to be 50 cells per $c / \omega_{p e}$ with 64 particles per cell which corresponds to a grid size of $\Delta x=0.02 c / \omega_{p e}$ and time step $\Delta t=0.012 \omega_{p e}^{-1}$. A small noise in terms of initial thermal velocity of $v_{\text {th }}=0.0001397 c$ in each of the two electron species has been introduced initially. Various choices of beam and background electron density have been chosen. In Fig. 2, we have chosen to show the snapshots of the evolution of the magnetic field and perturbed charge density at various times for a simulation run with a beam electron density of $0.1 n_{0}$ moving along $\hat{x}$ with a proper velocity of $0.9 c$ in a central region (from $y=10 c / \omega_{p e}$ to $y=15 c / \omega_{p e}$ ), i.e., with a 


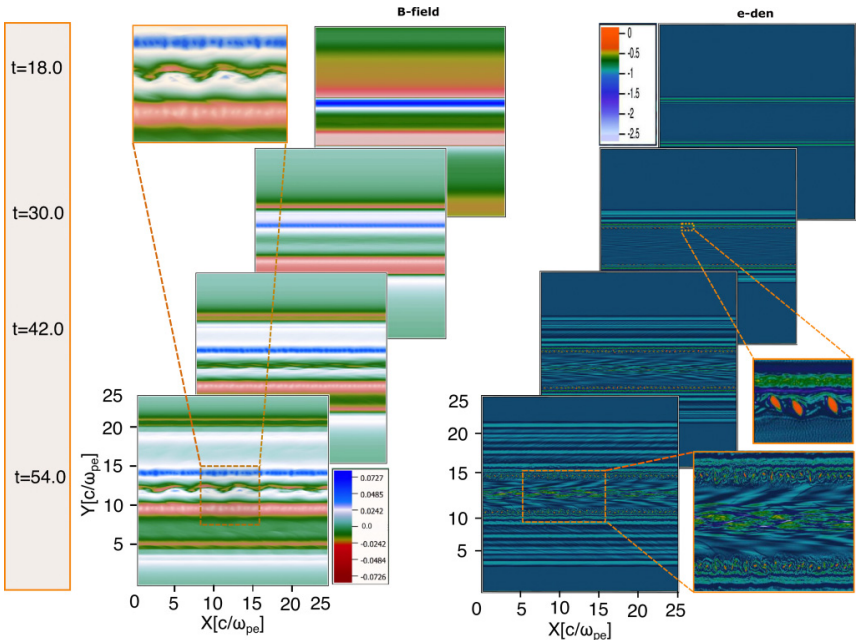

FIG. 2. Snapshots from 2D PIC simulations for magnetic field $B$ [in units of $\left(m c \omega_{p e} / e\right)$ ] and electron density evolution in time $t$ (in units of $\omega_{p e}^{-1}$ ) have been depicted (prepared by VAPOR $[19,20]$ ). The left and right series of subplots depict the spatiotemporal evolution of magnetic field $B_{z}$ and the electron density, respectively. At time $t=$ 18.0 the development of only long-scale magnetic field structures ( $B_{z}$ field with the opposite polarity) can be seen, and at $t=30$ the $\mathrm{KH}$ mode at the beam edge and a faint Weibel instability in the bulk appears. At $t=42.0$ and $t=54.0$ all three instabilities can be observed clearly. The zoomed region of the plots of $B_{z}$ at $t=54$ and perturbed electron density at $t=30$ has also been shown in the inset.

transverse extent of $5 c / \omega_{p e}$. In the same region, a shielding return current along $-\hat{x}$ of the background electrons with density $0.9 n_{0}$ has been taken to flow with a proper velocity of $0.1 c$. Here, $n_{0}$ is the density of background ions which are at rest everywhere. In the remaining region from $y=0$ to $10 \mathrm{c} / \omega_{p e}$ and 15 to $25 \mathrm{c} / \omega_{p e}$ electrons and ions both with density $n_{0}$ are at rest. Thus, the plasma is neutral everywhere with the electron density balancing the density of the background plasma ions. In the central beam region, the beam current is slightly uncompensated by the return shielding current. The movies uploaded as Supplemental Material [17] show the generation and evolution magnetic field for this particular configuration in 2D and 3D. We have also verified the results with PIC codes: EPOCH (see the Appendix, Fig. 7) and PICPSI [18]. The electromagnetic fluid simulations under the LCPFCT-Flux corrected transport algorithm for solving generalized continuity equation framework have also been carried out by us to validate the above findings.

From the plots of Fig. 2, it is clearly evident that there are three distinct phases of evolution. During the first phase from $t=0.12 \omega_{p e}^{-1}$ to $t=30 \omega_{p e}^{-1}$ perturbed $z$ components of the magnetic field along $\hat{z}$ (transverse to both flow and inhomogeneity) appear at the edges with opposite polarity. This magnetic field has no $x$ dependence and is a function of $y$ alone. The magnetic field perturbations are seen to grow with time and also expand in $y$ from the edges in both directions at the speed of light. The electron density perturbations, which also appear at the edge, on the other hand, remain confined at the edge during this phase. This first phase of the evolution thus can be characterized by the appearance of magnetic field perturbations with variations only along $\hat{y}$, the transverse (a)
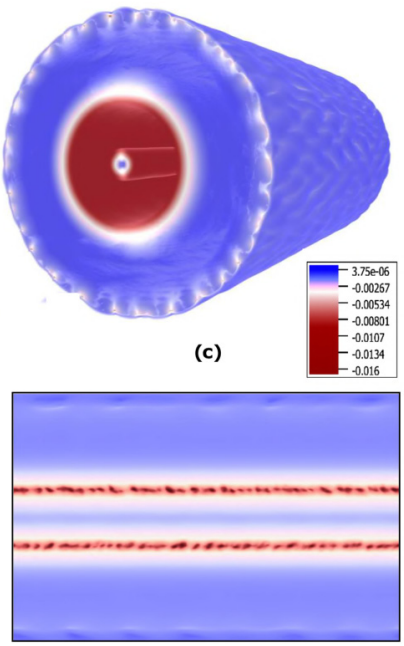

(b)

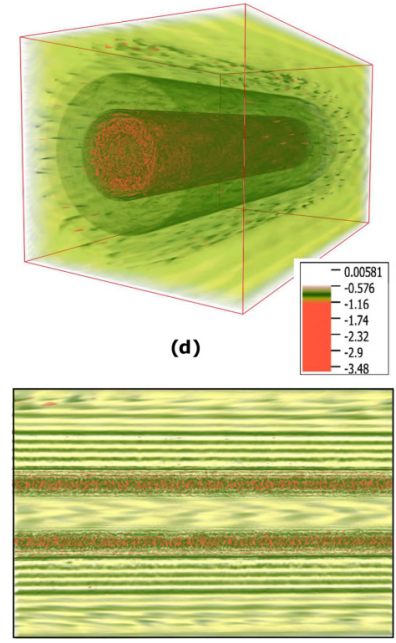

FIG. 3. Snapshots of 3D PIC simulation with OSIRIS (prepared by VAPOR $[19,20]$ ) at time $t=78.6$ in units of $\omega_{p e}^{-1}$. (a) and (b) depict a $3 \mathrm{D}$ volume rendering of the poloidal magnetic field and electron density, respectively. In (c) and (d) the cross-sectional cut along $z$ for the solid cylinder has been shown for the magnetic field and the electron density, respectively. In these 3D snapshots also all three instabilities, viz., long magnetic field structures and $\mathrm{KH}$ at the edge of the beam and Weibel in the bulk region of the beam, are clearly evident.

direction. This fits the analytical description very well. As the structures do not seem to vary with respect to the $x$ during the first phase, the 1D profiles along $y$ have been shown in Fig. 1(b) alongside the schematic for $E_{1 x}$. As predicted theoretically, $E_{1 x}$ shows minimal variation inside the beam region.

During the second phase from $t=30 \omega_{p e}{ }^{-1}$, KelvinHelmholtz (KH)-like perturbations appear at the edge of the current (Fig. 2). Around this time one can also observe the appearance of faint Weibel perturbations in the bulk central
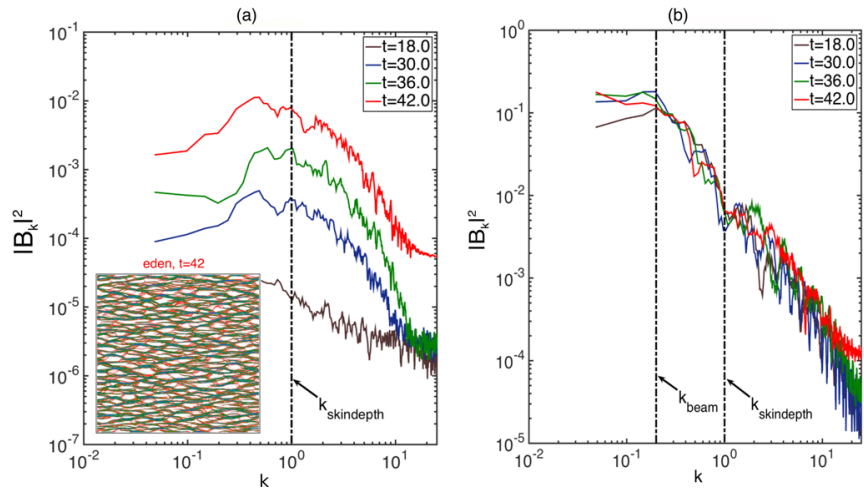

FIG. 4. Evolution of magnetic field spectra as a function of $k_{y}$ from PIC simulations with OSIRIS, (a) Periodic box simulations corresponding to an infinite beam-plasma system where the peak of the field spectra appears at the electron skin depth due to the Weibel mode and the nonlinear cascade towards the long scale can be seen to be very slow. (b) Finite beam-plasma system considered in the simulation. The spectral maximum is at the width of the beam right from the beginning. 


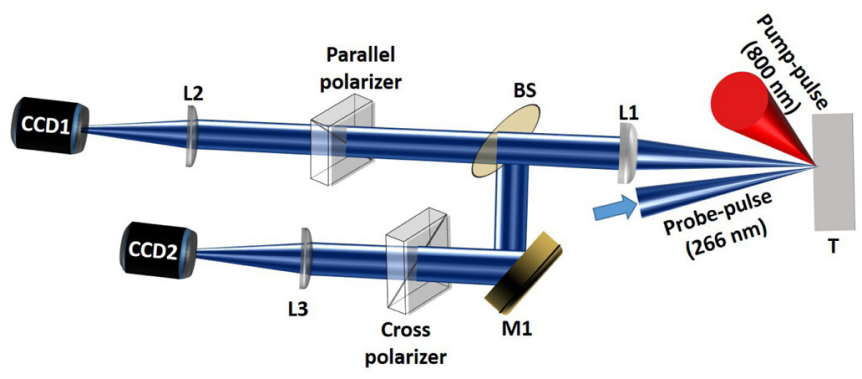

FIG. 5. Schematic (not to scale) of the experimental setup for measuring the megagauss magnetic fields at the target front. The 266-nm probe pulse reflects from the plasma and then is split using a nonpolarizing beam splitter (BS) and imaged onto CCDs through polarizers. L1, L2, L3: imaging lenses; M1: mirror; and T: aluminum-coated BK7 glass target.

region. Both the $\mathrm{KH}$ and the Weibel mode have variations along both $\hat{y}$ and $\hat{x}$ directions.

We have also carried out 3D simulations and in Fig. 3 we show the magnetic field and density snapshots for a finite solid cylindrical beam propagating in the plasma at $t=78.6 \omega_{p e}^{-1}$ at which the Weibel instability finally appears and all the three modes (viz., the finite beam, $\mathrm{KH}$, and Weibel) are clearly visible in the plot. These three distinct phases of development have been repeatedly observed in both 2D and 3D studies from a variety of simulations carried out with different PIC as well as fluid codes.

Figure 4 presents a comparison of the evolution of the magnetic field spectra for the periodic system as well as the finite beam case. It is clear from the figure that for the periodic case, the peak of spectral power appears initially at the electron skin depth scale. In this case, the spectral power only subsequently cascades towards longer scales via an inverse cascade mechanism which is considerably slow. On the other hand, for the finite beam case, it can be clearly observed that the spectral peak appears at the beam size right from the very beginning.

We now present experiments carried out at the Tata Institute of Fundamental Research, Mumbai using a 20-TW Ti:sapphire laser. A schematic of the experiment is shown in Fig. 5. The laser delivers $30-\mathrm{fs}, 800-\mathrm{nm}$ pulses at $10 \mathrm{~Hz}$. A $p$-polarized pump laser with $120 \mathrm{~mJ}$ energy was focused on an aluminum-coated BK7 glass target with an $f / 3$ off-axis parabola. The resulting peak intensity was $3 \times 10^{18} \mathrm{~W} / \mathrm{cm}^{2}$. A probe pulse at $266 \mathrm{~nm}$ was time delayed with respect to the pump pulse. The intensity of the probe pulse was adjusted to be $10^{11} \mathrm{~W} / \mathrm{cm}^{2}$. The third harmonic probe can penetrate up to densities that are nine times that of the critical density for the 800-nm pump [21]. A magneto-optic Cotton-Mouton polarimetry setup $[4,21]$ was utilized to spatially resolve ellipticity. The induced magnetic field is inferred from the ellipticity data [22]. The power spectra have been calculated as per Mondal et al. [4] and shown to peak at the focal spot of the laser pulse right from the very beginning.

In laboratory laser-plasma experiments the electron beam width would always be finite, typically commensurate with the dimension of the laser focal spot. Also, there would typically be a mismatch between the forward and reverse shielding

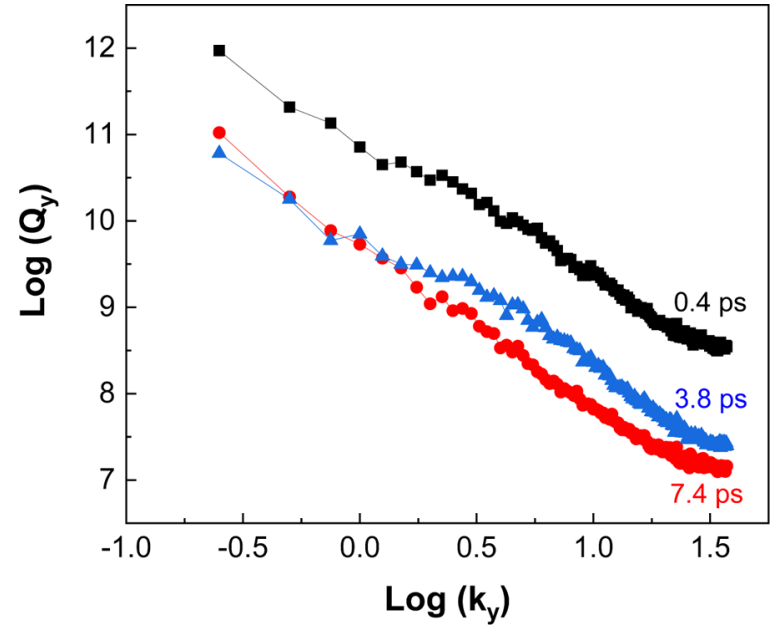

FIG. 6. Power spectrum of magnetic field spatial profiles measured at different pump-probe delays. The magnetic-energy spectra show the formation of long-scale magnetic field structures (of the laser focal width which is much longer than the electron skin depth) even at very early times of $t=0.4 \mathrm{ps}, t=3.8 \mathrm{ps}$, and $t=7.4 \mathrm{ps}$. Here, $Q_{y}=\int\left|B_{z}\left(k_{x}, k_{y}\right)\right|^{2} d k_{x}$. The unit of the wave number here is $(\mu \mathrm{m})^{-1}$.

currents. The finite beam mechanism is obviously operative in laser-plasma experiments, as shown by the magnetic field power spectra. The power is clearly higher at scales much longer than the electron skin depth even at very early times $[4,6]$. Figure 6 shows a plot of the experimentally observed magnetic field power spectra at even earlier times (viz., 0.4, 3.8 , and $7.4 \mathrm{ps}$ ). In this case, too, the power is higher at longer scales. Thus, experiments provide compelling evidence for the existence of long-scale magnetic field structures.

The choice of a periodic beam-plasma system considered in earlier studies [23] is based on an inherent assumption that the boundary effects due to the finite beam size would merely have a small incremental impact. This assumption, however, turns out to be incorrect as we have shown above with PIC as well as two-fluid simulations for a beam with a finite transverse extent. An entirely different mechanism associated with the finite size of the beam appears which generates magnetic fields at the scale of the beam size right from the very beginning. Second, the sheared electron flow configuration at the two edges of the finite beam is seen to be susceptible to Kelvin-Helmholtz instability [24,25]. The much investigated Weibel appears (a) only in the bulk region of the beam and (b) at a much later stage. Thus, there are three sources of the generation of magnetic fluctuations in a finite beam-plasma system: (a) the mechanism of long-scale generation via radiative leakage that we demonstrate, (b) the KH instability operating at the edge, and finally (c) the Weibel destabilization process occurring in the bulk region. The characteristic features of the observation in simulations [both PIC and fluid (LCPFCT [26]] match with the analytic calculation of the growing mode, demonstrated earlier in the paper. Furthermore, the experimental data shown in Fig. 6 and elsewhere [4] where the magnetic field appears at the laser spot size (much larger than the skin depth) at a very early stage, can be explained only by this mechanism. The conventional Weibel destabilization 
route of the beam-plasma system cannot account for such an effect.

While the $\mathrm{KH}$ and the Weibel modes are well known and have been discussed extensively in the literature, the long-scale magnetic field generated by the radiative leakage from the boundaries has yet to be shown in simulations and described. A recent work by Grismeyer et al. [12] reports the formation of long scales in the longitudinal beam propagation direction. This would correspond to observing high power at long scales in the longitudinal power spectrum of the magnetic field. The authors have attributed this to KelvinHelmholtz shear flow instabilities. In total contrast, our work shows a significant magnetic power density at long scales in the transverse spectrum. The infinite periodic approximation in previous work prevents the capture of the qualitatively different result that we have shown, simply because it does not permit accounting for radiative leakages at the boundary (there are no boundaries in that approach).

The implications of this method of magnetic field generation need to be examined in different contexts. For instance, it is likely that the finite-size jets emanating from astrophysical objects are susceptible to this particular instability. This work also suggests that finite-size considerations in many other systems need to be investigated afresh to unravel effects which might have been overlooked so far.

Finally, we present some general remarks. Homogeneity and infinite extent are idealizations that permeate all physical models, as they simplify descriptions resulting from the uniformity and reduced dimensionality of the problem. Real systems, however, are finite and deviations from idealization can lead to other physical effects, a lesson relearned from time to time. Some examples of finite-size boundaries leading to different effects, cutting across various research areas, are the Casimir effect $[27,28]$ that results in an attractive force between two plates due to vacuum fluctuations, an einzel lens [29,30] which enables focusing of charged particles using fringing fields at capacitor edges, surface plasmon modes [31] distinct from bulk modes, discrete eigenmodes in waveguides, etc. The present study demonstrates another outstanding example in this context.

\section{ACKNOWLEDGMENTS}

The authors thank the OSIRIS Consortium consisting of UCLA and IST (Lisbon, Portugal), for providing access to the OSIRIS-4.0 framework which is the work supported by NSF ACI-1339893. A.D. also wishes to thank Prof. Warren Mori (UCLA) for discussions. A.D. and A.K. would like to acknowledge discussions with Dr. Kevin M. Schoeffler, IST, Lisbon. A.D. acknowledges her Core Research Grant No. CRG/2018/000624 of Department of Science and Technology (DST), Government of India. A.D. and G.R.K. acknowledge support from their respective J. C. Bose Fellowship grants (JCB-000055/2017 and JCB-037/2010) from the Science and Engineering Research Board (SERB), Government of India. A part of the research has also been supported by the S-level funding 15H05751, JSPS Japan.

\section{APPENDIX}

The simulation results from the EPOCH simulations also confirm the appearance of a long-scale magnetic field much before conventional beam-plasma instabilities such as KelvinHelmholtz (KH), Weibel instabilities, etc., as shown in Fig. 7.

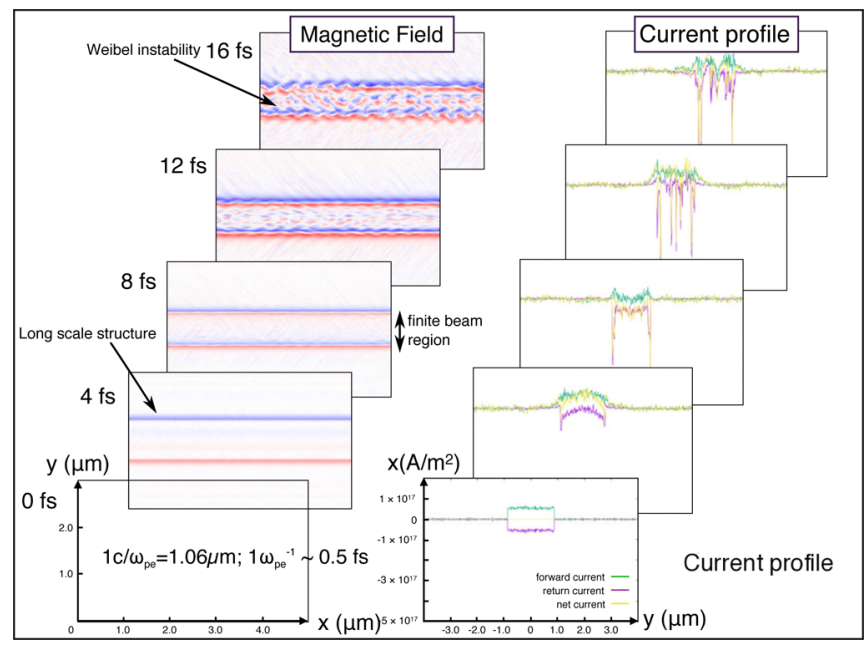

FIG. 7. Temporal evolution of the results from 2D PIC simulations using the EPOCH code for magnetic field $B_{z}$ and electron current evolution. It is evident from the left subplot of the magnetic field that at up to time $t=8.0 \mathrm{fs}=16 \omega_{p e}^{-1}$, the development of only long-scale magnetic field structures ( $B_{z}$ field with the opposite polarity) can be seen, and at $t=12 \mathrm{fs}=24 \omega_{p e}$, the KH mode at the beam edge and a faint Weibel instability in the bulk appears which grows with time as shown at $t=16 \mathrm{fs}=32 \omega_{p e}$.
[1] K. B. Wharton, S. P. Hatchett, S. C. Wilks, M. H. Key, J. D. Moody, V. Yanovsky, A. A. Offenberger, B. A. Hammel, M. D. Perry, and C. Joshi, Experimental Measurements of Hot Electrons Generated by Ultraintense Laser-Plasma Interactions on Solid-Density Targets, Phys. Rev. Lett. 81, 822 (1998).

[2] K. A. Brueckner and S. Jorna, Laser-driven fusion, Rev. Mod. Phys. 46, 325 (1974).

[3] R. Betti and O. A. Hurricane, Inertial-confinement fusion with lasers, Nat. Phys. 12, 435 (2016).

[4] S. Mondal, V. Narayanan, W. J. Ding, A. D. Lad, B. Hao, S. Ahmad, W. M. Wang, Z. M. Sheng, S. Sengupta, P. Kaw, A.
Das, and G. R. Kumar, Direct observation of turbulent magnetic fields in hot, dense laser produced plasmas, Proc. Natl. Acad. Sci. U.S.A. 109, 8011 (2012).

[5] B. A. Remington, D. Arnett, R. Paul, Drake, and H. Takabe, Modeling astrophysical phenomena in the laboratory with intense lasers, Science 284, 1488 (1999).

[6] G. Chatterjee, K. M. Schoeffler, P. Kumar Singh, A. Adak, A. D. Lad, S. Sengupta, P. Kaw, L. O. Silva, A. Das, and G. R. Kumar, Magnetic turbulence in a table-top laser-plasma relevant to astrophysical scenarios, Nat. Commun. 8, 15970 (2017). 
[7] P. K. Kaw, Nonlinear laser-plasma interactions, Rev. Mod. Plasma Phys. 1, 2 (2017).

[8] E. S. Weibel, Spontaneously Growing Transverse Waves in a Plasma Due to an Anisotropic Velocity Distribution, Phys. Rev. Lett. 2, 83 (1959).

[9] A. Kumar, C. Shukla, A. Das, and P. Kaw, Energy principle for excitations in plasmas with counterstreaming electron flows, AIP Adv. 8, 055213 (2018).

[10] F. Pegoraro, S. V. Bulanov, F. Califano, and M. Lontano, Nonlinear development of the Weibel instability and magnetic field generation in collisionless plasmas, Phys. Scr. 1996, 262 (1996).

[11] J. C. Adam, A. Héron, and G. Laval, Dispersion and Transport of Energetic Particles due to the Interaction of Intense Laser Pulses with Overdense Plasmas, Phys. Rev. Lett. 97, 205006 (2006).

[12] T. Grismayer, E. P. Alves, R. A. Fonseca, and L. O. Silva, dc-Magnetic-Field Generation in Unmagnetized Shear Flows, Phys. Rev. Lett. 111, 015005 (2013).

[13] C. Ruyer, L. Gremillet, and G. Bonnaud, Weibel-mediated collisionless shocks in laser-irradiated dense plasmas: Prevailing role of the electrons in generating the field fluctuations, Phys. Plasmas 22, 082107 (2015).

[14] D. J. Stark, T. Toncian, and A. V. Arefiev, Enhanced Multi-MeV Photon Emission by a Laser-Driven Electron Beam in a SelfGenerated Magnetic Field, Phys. Rev. Lett. 116, 185003 (2016).

[15] M. Miyagi, Bending losses in hollow and dielectric tube leaky waveguides, Appl. Opt. 20, 1221 (1981).

[16] D. Botez, L. Mawst, P. Hayashida, G. Peterson, and T. J. Roth, High-power, diffraction-limited-beam operation from phaselocked diode-laser arrays of closely spaced leaky waveguides (antiguides), Appl. Phys. Lett. 53, 464 (1988).

[17] See Supplemental Material at http://link.aps.org/supplemental/ 10.1103/PhysRevResearch.2.033405 for the movie which depicts time evolution of magnetic field structures in three dimensional simulations at various $2-D$ planes. It clearly show the formation of long scale magnetic field at very early stage which is followed up only later by Kelvin Helmholtz mode appearing at the edge.

[18] C. Shukla, A. Das, and K. Patel, Effect of finite beam width on current separation in beam plasma system: Particle-in-Cell simulations, arXiv:1508.07701.

[19] J. Clyne, P. Mininni, A. Norton, and M. Rast, Interactive desktop analysis of high resolution simulations: Application to turbulent plume dynamics and current sheet formation, New J. Phys. 9, 301 (2007).
[20] J. Clyne and M. Rast, A prototype discovery environment for analyzing and visualizing terascale turbulent fluid flow simulations, Proc. SPIE 5669, 284 (2005).

[21] G. Chatterjee, P. K. Singh, A. Adak, A. D. Lad, and G. R. Kumar, High-resolution measurements of the spatial and temporal evolution of megagauss magnetic fields created in intense short-pulse laser-plasma interactions, Rev. Sci. Instrum. 85, 013505 (2014).

[22] A. S. Sandhu, A. K. Dharmadhikari, P. P. Rajeev, G. R. Kumar, S. Sengupta, A. Das, and P. K. Kaw, Laser-Generated Ultrashort Multimegagauss Magnetic Pulses in Plasmas, Phys. Rev. Lett. 89, 225002 (2002).

[23] Y. Sentoku, K. Mima, Z. M. Sheng, P. Kaw, K. Nishihara, and K. Nishikawa, Three-dimensional particle-in-cell simulations of energetic electron generation and transport with relativistic laser pulses in overdense plasmas, Phys. Rev. E 65, 046408 (2002).

[24] A. Das and P. Kaw, Nonlocal sausage-like instability of current channels in electron magnetohydrodynamics, Phys. Plasmas 8 , 4518 (2001).

[25] C. Shukla, A. Das, and K. Patel, Particle-in-cell simulation of two-dimensional electron velocity shear driven instability in relativistic domain, Phys. Plasmas 23, 082108 (2016).

[26] J. P. Boris, A. M. Landsberg, E. S. Oran, and J. H. Gardner, LCPFCT - A flux-corrected transport algorithm for solving generalized continuity equations, NRL Memorandum Report No. 93-7192, Naval Research Laboratory, Washington, 1993 (unpublished).

[27] H. B. G. Casimir and D. Polder, The influence of retardation on the London-van der Waals forces, Phys. Rev. 73, 360 (1948).

[28] H. B. G. Casimir, On the attraction between two perfectly conducting plates, H. B. G. Indag. Math. 10, 261 (1948); Kon. Ned. Akad. Wetensch. Proc. 51, 793 (1948), https://www.dwc. knaw.nl/DL/publications/PU00018547.pdf; Front. Phys. 65, 342 (1987).

[29] H. Liebl, Applied Charged Particle Optics (Springer, Berlin, 2008).

[30] R. Feynman, R. B. Leighton, and M. Sands, The Feynman Lectures on Physics 2 (Addison-Wesley, Reading, MA, 1964).

[31] E. A. Stern and R. A. Ferrell, Surface plasma oscillations of a degenerate electron gas, Phys. Rev. 120, 130 (1960).

Correction: The omission of a support statement in the Acknowledgments has been fixed. 